\title{
Regular and transitory showers of comet C/1979 Y1 (Bradfield)
}

\author{
M. Hajduková, Jr. ${ }^{1}$ and L. Neslušan ${ }^{2}$ \\ 1 Astronomical Institute, Slovak Academy of Science, Dúbravská cesta 9, 84504 Bratislava, Slovakia \\ e-mail: astromia@savba.sk \\ 2 Astronomical Institute, Slovak Academy of Science, 05960 Tatranská Lomnica, Slovakia \\ e-mail: ne@ta3.sk
}

Received 17 February 2017 / Accepted 17 May 2017

\begin{abstract}
Aims. We intend to map the whole meteor complex of the long-period comet C/1979 Y1 (Bradfield), which is a proposed parent body of the July Pegasids, No. 175 in the list of meteor showers established by the Meteor Data Center (MDC) of the International Astronomical Union (IAU).

Methods. For five perihelion passages of the parent comet in the past, we model associated theoretical stream, its parts, each consisting of 10000 test particles, and follow the dynamical evolution of these parts up to the present. Subsequently, we analyze the mean orbital characteristics of those particles of the parts that approach the Earth's orbit and, thus, create a shower or showers. The showers are compared with their observed counterparts separated from photographic, radio, and several video databases.

Results. The modeled stream of C/1979 Y1 approaches the Earth's orbit in two filaments that correspond to two regular (annual) showers. We confirm the generic relationship between the studied parent comet and 175 July Pegasids. The other predicted shower is a daytime shower with the mean radiant situated symmetrically to the July Pegasids with respect to the apex of the Earth's motion. This shower is not in the IAU MDC list, but we separated it from the Cameras-for-Allsky-Meteor-Surveillance (CAMS) and SonotaCo video data as a new shower. We suggest naming it $\alpha$-Microscopiids. The stronger influence of the Poynting-Robertson drag deflects the stream away from the Earth's orbit in those sections that correspond to the July Pegasids and the predicted daytime shower, but it makes the stream cross the Earth's orbit in other sections. Corresponding showers are, however, only expected to survive during a limited period and to consist of particles of sizes in a narrow interval. We identified one of these "transitory" filaments to the $104 \gamma$-Bootids in the IAU MDC list of meteor showers.
\end{abstract}

Key words. comets: individual: C/1979 Y1 (Bradfield) - meteorites, meteors, meteoroids

\section{Introduction}

The orbit of the parent body of the meteoroid stream is commonly situated within the orbital corridor of the respective stream. However, the perturbations of major planets can change the initial orbits of a part of the stream and alternative corridors of orbits can be formed. If more than a single corridor of a given stream passes through the Earth's orbit, we observe several meteor showers associated with the same parent body.

The alteration of the initial orbital corridor can be revealed by modeling the stream and studying its dynamical evolution for a suitably long period. Meteoroid streams of several parent bodies were studied with the help of various stream models (e.g., Asher \& Emel'yanenko 2002; Lyytinen \& Jenniskens 2003; Wiegert et al. 2005; Vaubaillon et al. 2005a,b; Vaubaillon \& Jenniskens 2007; Kaňuchová \& Neslušan 2007, Asher 2008, Jenniskens \& Vaubaillon (2010); Vereš et al. 2011; Jopek \& Williams 2013; Sekhar \& Asher 2013; 2014a,b; Neslušan et al. 2013a,b; Neslušan \& Hajduková 2014; Babadzhanov et al. 2008, 2013, 2015a,b; Jakubík \& Neslušan 2015; Kasuga \& Jewitt 2015; Kokhirova \& Babadzhanov 2015; Kornoš et al. 2015; Rudawska \& Vaubaillon 2015; Ryabova 2007, 2016; Tomko \& Neslušan 2012, 2016; Micheli et al. 2016; Abedin et al. 2015, 2017)
In our work modeling streams, we attempt not only to find new parent bodies of known meteor showers, but also to predict new meteor showers associated with as many as possible known periodic comets. Most of our previous studies deal with parent bodies on short-period orbits. In this contribution, we focus our attention on the long-period comet C/1979 Y1 (Bradfield). We model and study the dynamical evolution of the stream related to this comet, which allows us to map its whole meteor shower complex.

The association of comet C/1979 Y1 to the July Pegasids has been suggested by several authors (e.g., Rendtel et al. 1995; Jenniskens 2006, Table 7, \#175 on page 715). The July Pegasid shower (No. 175 in the IAU MDC list of established showers) is a weak meteor shower with a short activity period. The shower was observed by the IMO Video Meteor Network (Rendtel et al. 1995; Molau \& Rendtel 2009), the SonotaCo Network (Ueda 2012), the Croatian Meteor Network (Andreić et al. 2013), and by the Cameras for Allsky Meteor Surveillance (Gural 2011; Jenniskens et al. 2011; Holman \& Jenniskens 2012). The latter authors also proposed the $\mathrm{C} / 1771 \mathrm{~A} 1$ as a possible parent comet, suggesting that both $\mathrm{C} / 1979 \mathrm{Y} 1$ and $\mathrm{C} / 1771 \mathrm{~A} 1$ may be the same comet or they may have originated from a common progenitor at the time of the formation of the July Pegasid shower. 
As in several previous papers (e.g., Neslušan et al. 2013b), we use the words "complex" and "stream" as synonyms referring to the whole structure of meteoroids released from the parent body. The complex consists of several "filaments". If the particles of a filament hit the Earth, they can cause the meteor "shower" corresponding to the filament.

\section{The orbit of the parent comet}

In our study, we consider the orbit of comet C/1979 Y1 with the orbital elements published in the JPL small-body browser (Giorgini et al. (1996) ${ }^{1}$. Specifically, the orbit referred to epoch 1980 January 25.0 (JDT $=2444263.5$ ) has elements: $q=0.5452748296 \pm 0.000030079 \mathrm{au}, e=$ $0.987952184 \pm 0.000098692, a=45.25923 \pm 0.37324$ au $\omega=257.60493676^{\circ} \pm 0.0054013^{\circ}, \Omega=103.21615886^{\circ} \pm$ $0.00092632^{\circ}, \quad i=148.60242336^{\circ} \pm 0.00030014^{\circ}$, and $=$ 2444229.10764317 (1979 December 21.60764317). Hereinafter, this orbit is referred to as the nominal orbit. The orbital period of the comet in the nominal orbit is $304.5 \mathrm{yrs}$.

To follow the past evolution of this orbit, including the uncertainty of its determination, we create 100 clones, using the method by Chernitsov et al. (1998). If the orbital elements of the nominal orbit are written in the form of covariant $6 \times 1$ matrix $y_{\mathrm{o}}$, the corresponding covariant matrix with the elements of the orbit of the $j$ th clone, $y_{j}$, can be calculated as

$y_{j}=y_{\mathrm{o}}+\mathbf{A} \eta^{T}$,

where $\mathbf{A}$ is such a triangle matrix that the product $\mathbf{A} \mathbf{A}^{T}$ equals the covariance matrix related to the process of nominal-orbit determination (the covariance matrix can again be found on the website of the JPL browser). Since we have six elements, $\mathbf{A}$ is a $6 \times 6$ matrix. $\eta$ is a $1 \times 6$ matrix with each element; $\eta_{i}$ being a random number from the interval $(0,1)$, that is $0 \leq \eta_{i} \leq 1 . \eta^{T}$ is its covariant form.

We integrated the nominal orbit and all the orbits of the clones backward in time, down to 80000 yr. The past evolution of the orbits of the clones and the nominal orbit is shown in Fig. 1.

The minimum distance of both pre-perihelion and postperihelion arcs of C/1979 Y1's orbit from the Earth's orbit is shown in Fig. 2. Both orbital arcs approach the orbit of our planet at present relatively closely, within $0.15 \mathrm{au}$, and, thus, both could induce a meteor shower. The positions of the nodes of the nominal orbit during the last 40 millennia can be seen in Fig. 3 .

\section{Modeling the stream}

To study the theoretical stream of comet C/1979 Y1, we use the procedure suggested by Neslušan (1999), which was slightly modified and described in more detail by Tomko \& Neslušan (2012). The orbit of the parent body is integrated backward in time, to the moment of the body's perihelion passage, which happened closest to an arbitrarily chosen time $t_{\mathrm{ev}}$. In the perihelion, a cloud of 10000 test particles around the parent body is modeled and all this assembly is integrated forward in time, up to the present. At the end of the integration, the particles moving in the orbits that approach the Earth's orbit within 0.05 au are selected and used to predict the mean characteristics of the expected shower. A search for a corresponding shower is then made in the meteor database(s) available. A brief summary of the

\footnotetext{
1 http://ssd.jpl.nasa.gov/sbdb.cgi
}

method can also be found in papers by Neslušan et al. (2013b), Jakubík \& Neslušan (2015), and others. Since the method has been described several times, we do not repeat its more detailed description in the present paper.

To integrate the orbits, we use integrator RA15 (Everhart 1985) within the software package MERCURY (Chambers 1999). The gravitational perturbations of eight planets, Mercury to Neptune, are taken into account.

In the simulations performed, the acceleration due to the Poynting-Robertson (P-R) effect is considered. When speaking about the P-R effect acting on meteoroid particles, we mean an action of radial electromagnetic radiation pressure as well as the velocity-dependent effects. In contrast to all the papers we have co-authored in the past, in this work we include the effects of solar wind (in addition to radiation) in our calculation of the acceleration. Specifically, here we use the improved formulas derived by Klačka (2014). Assuming constant solar wind, the radial and transversal components of the acceleration due to these effects are

$$
\begin{aligned}
& a_{r}=\beta \frac{G M_{\odot}}{r^{2}}\left[1-\left(2+\frac{\eta_{1}+\eta_{2}}{Q_{\mathrm{pr}}^{\prime}}\right) \frac{v_{r}}{c}\right], \\
& a_{t}=-\beta \frac{G M_{\odot}}{r^{2}}\left(1+\frac{\eta_{2}}{Q_{\mathrm{pr}}^{\prime}}\right) \frac{v_{t}}{c},
\end{aligned}
$$

and the perpendicular component is zero. In Eqs. (2) and (3), $\beta$ is the ratio of the acceleration due to both effects to gravitational acceleration by the Sun, $G$ is the gravitational constant, $M_{\odot}$ is the mass of the Sun, $r$ is the heliocentric distance of the meteoroid particle, $v_{r}$ and $v_{t}$ are the radial and transversal components of its heliocentric velocity, and $c$ is the speed of light in a vacuum. Component $v_{t}$ is assumed to lay in the particle's orbital plane (therefore, the perpendicular component of the velocity vector must be zero). Further, $Q_{\mathrm{pr}}^{\prime}$ is the dimensionless efficiency factor of the radiation pressure averaged over the solar spectrum, and $\eta_{1}$ and $\eta_{2}$ are the parameters characterizing the solar wind.

In the solar system, the recommended values for $\eta_{1}$ and $\eta_{2}$ are $\eta_{1}=1.1$ and $\eta_{2}=1.4$. We assume the efficiency factor $Q_{\mathrm{pr}}^{\prime}=$ 1 . The relation between the parameter $\beta$ and the efficiency factor $Q_{\mathrm{pr}}^{\prime}$ is

$$
\beta=576 \frac{Q_{\mathrm{pr}}^{\prime}}{\rho R},
$$

where $\rho$ is the mean mass density of a meteoroid particle in kilograms per cubic meter and $R$ is its average radius in microns.

In the formula for the calculation of $\beta$, its dependence on the size (geometrical cross-section), density, light-scattering effectivity (then on albedo and light-absorption ability), and mass of the particles is seen. In our case, these properties are rather uncertain, therefore we could calculate the value of $\beta$ with a large uncertainty. Instead of such a calculation, we regard $\beta$ as a free parameter and search, as far as possible, for its value to achieve the best agreement between the predicted and observed characteristics of found filaments of C/1979 Y1's complex.

We create a series of models for various combinations of specific values of evolutionary time $t_{\mathrm{ev}}$ and parameter $\beta$. Hence, a given model does not represent a whole stream, since the real stream consists of the particles of various sizes and densities and, therefore, parameter $\beta$ ranges in a wider interval of values. Also, the particles are released at various times in reality, therefore their evolutionary time is different and can acquire a value from a wider interval. The sufficient combinations of $t_{\mathrm{ev}}$ and $\beta$ are found by comparing predicted and observed showers. 

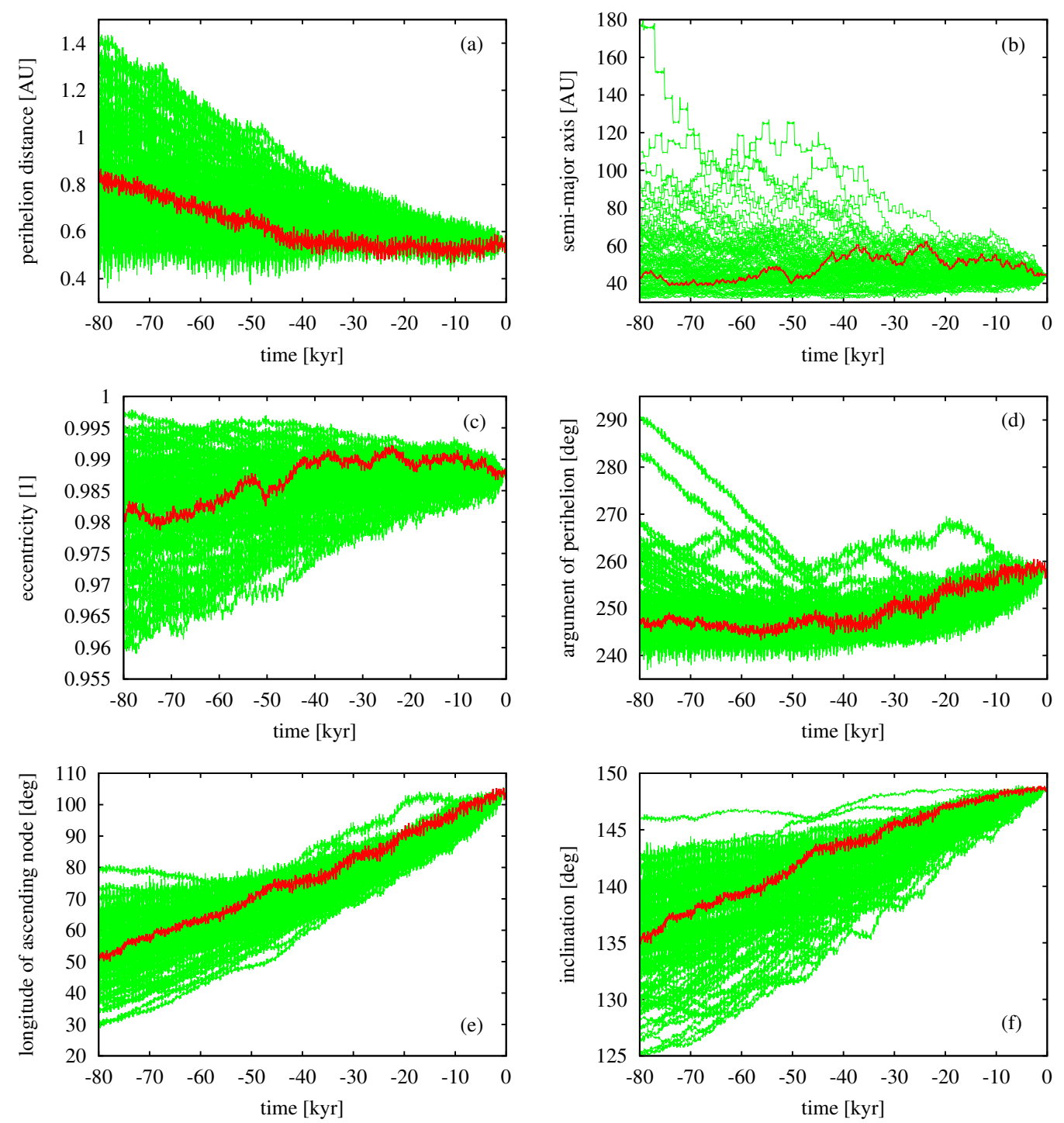

Fig. 1. Behavior of perihelion distance (plot $a$ ), semi-major axis $(b)$, eccentricity $(c)$, argument of perihelion $(d)$, longitude of ascending node $(e)$, and inclination to the ecliptic $(f)$ of the nominal orbit of comet C/1979 Y1 (red curve) and the orbits of its clones (green curves) mapping the uncertainty of the determination of nominal orbit. The evolution is reconstructed backward for $80000 \mathrm{yr}$.

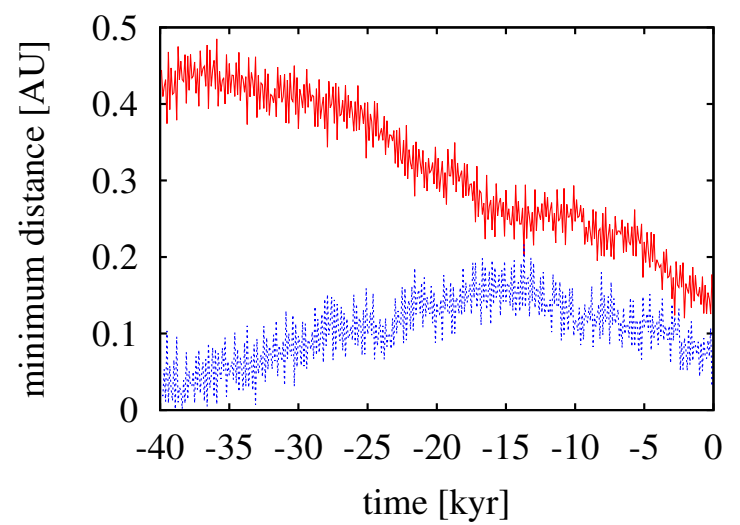

Fig. 2. Evolution of the minimum distance between the orbital arcs of comet C/1979 Y1 and Earth from a time 40000 yr before the present to the present. The minimum distance of post-perihelion (pre-perihelion) arc is shown by the red solid (blue dashed) curve.



Fig. 3. Positions of the orbital nodes of comet C/1979 Y1 during the last $40000 \mathrm{yr}$. The green circle indicates the orbit of the Earth. The red dots outside the Earth's orbit (blue dots inside the Earth's orbit) show the positions of ascending (descending) node. 
Table 1. Mean geophysical characteristics of the predicted annual meteor showers.

\begin{tabular}{|c|c|c|c|c|c|c|c|c|c|}
\hline$t_{\mathrm{ev}} ;$ Ref. & $\beta$ & $\lambda_{\odot}$ & $\alpha$ & $\delta$ & $V_{\mathrm{g}}$ & $V_{\mathrm{h}}$ & $\gamma$ & $N_{\mathrm{s}}$ & $D_{\lim }$ \\
\hline \multicolumn{10}{|c|}{ Filament F1 - 175 July Pegasids } \\
\hline \multirow[t]{2}{*}{5} & 0.00001 & $111.0 \pm 3.1$ & $348.5 \pm 2.2$ & $11.2 \pm 0.4$ & $64.0 \pm 0.2$ & $41.6 \pm 0.1$ & 116 & 49 & 0.08 \\
\hline & 0.001 & $108.4 \pm 2.3$ & $346.7 \pm 1.6$ & $10.9 \pm 0.3$ & $64.1 \pm 0.2$ & $41.5 \pm 0.1$ & 115 & 19 & 0.06 \\
\hline \multirow[t]{2}{*}{10} & 0.00001 & $114.3 \pm 4.0$ & $351.4 \pm 3.2$ & $11.9 \pm 0.8$ & $63.9 \pm 0.3$ & $41.6 \pm 0.1$ & 116 & 943 & 0.15 \\
\hline & 0.001 & $111.8 \pm 3.5$ & $349.4 \pm 2.7$ & $11.4 \pm 0.6$ & $64.1 \pm 0.3$ & $41.6 \pm 0.1$ & 116 & 456 & 0.13 \\
\hline \multirow[t]{2}{*}{20} & 0.00001 & $117.6 \pm 5.6$ & $354.3 \pm 4.6$ & $12.7 \pm 1.3$ & $63.8 \pm 0.4$ & $41.5 \pm 0.1$ & 117 & 778 & 0.20 \\
\hline & 0.001 & $115.3 \pm 4.8$ & $352.4 \pm 3.9$ & $12.2 \pm 1.1$ & $64.0 \pm 0.3$ & $41.6 \pm 0.1$ & 116 & 443 & 0.19 \\
\hline \multirow[t]{2}{*}{40} & 0.00001 & $115.8 \pm 6.6$ & $352.9 \pm 5.4$ & $12.7 \pm 1.7$ & $63.9 \pm 0.4$ & $41.5 \pm 0.1$ & 116 & 450 & 0.21 \\
\hline & 0.001 & $114.2 \pm 7.5$ & $351.8 \pm 6.1$ & $12.4 \pm 1.7$ & $64.1 \pm 0.4$ & $41.5 \pm 0.1$ & 116 & 307 & 0.29 \\
\hline \multirow[t]{2}{*}{80} & 0.00001 & $111.6 \pm 11.0$ & $349.1 \pm 9.3$ & $12.5 \pm 3.0$ & $63.9 \pm 0.5$ & $41.5 \pm 0.1$ & 115 & 1171 & 0.39 \\
\hline & 0.001 & $109.3 \pm 12.8$ & $347.5 \pm 10.7$ & $12.0 \pm 3.3$ & $64.1 \pm 0.5$ & $41.5 \pm 0.1$ & 115 & 966 & 0.41 \\
\hline \multicolumn{10}{|c|}{ July Pegasids separated from CAMS database in this work } \\
\hline & & $110.8 \pm 5.5$ & $350.0 \pm 4.6$ & $11.7 \pm 1.6$ & $64.1 \pm 1.1$ & $41.0 \pm 1.0$ & 114 & 81 & 0.17 \\
\hline \multicolumn{10}{|c|}{ July Pegasids in the IAU MDC list } \\
\hline Jennisken & $(2006)$ & 107.5 & $340 \pm 0.6$ & $15 \pm 0.2$ & 61.3 & & 118 & & \\
\hline Molau \& & Rendtel (2009) & 108 & $347.2 \pm 0.9$ & $11.1 \pm 0.2$ & 68.1 & & 114 & 591 & \\
\hline Ueda $(20$ & & 110.9 & $349.6 \pm 0.71$ & $11.3 \pm 0.17$ & 63.9 & & 115 & 63 & \\
\hline Holman \& & Jenniskens (2012) & 109 & 348.8 & 11.1 & 64.5 & & 114 & & \\
\hline Jennisken & et al. (2016a) & 108 & $347.2 \pm 0.9$ & $10.0 \pm 0.39$ & 64.0 & & 115 & 104 & \\
\hline \multicolumn{10}{|c|}{ Southern alpha Pegasids in the IAU MDC list } \\
\hline \multicolumn{2}{|c|}{ Andreić et al. (2013) } & 112.0 & 351 & 11.7 & 63.9 & & 115 & 93 & \\
\hline \multicolumn{10}{|c|}{ July gamma Pegasids in the IAU MDC list } \\
\hline \multicolumn{2}{|c|}{ Rudawska \& Jenniskens (2014) } & 120.8 & 358.6 & 14.2 & 62.6 & & 116 & 8 & \\
\hline \multicolumn{2}{|c|}{ Kornoš et al. (2014b) } & 120.5 & 357.1 & 13.3 & 62.31 & & 117 & 12 & \\
\hline \multirow{4}{*}{5} & & & Filam & it F2 & & & & & \\
\hline & 0.00001 & $292.9 \pm 2.1$ & $224.1 \pm 1.5$ & $-30.0 \pm 0.5$ & $65.2 \pm 0.2$ & $41.9 \pm 0.0$ & 63 & 6 & 0.04 \\
\hline & 0.0001 & $293.0 \pm 1.3$ & $224.0 \pm 1.0$ & $-29.9 \pm 0.4$ & $65.2 \pm 0.1$ & $41.8 \pm 0.0$ & 63 & 3 & 0.02 \\
\hline & 0.001 & - & - & - & - & - & - & 0 & - \\
\hline \multirow[t]{2}{*}{10} & 0.00001 & $297.0 \pm 4.2$ & $228.4 \pm 3.7$ & $-31.5 \pm 1.4$ & $65.3 \pm 0.3$ & $42.0 \pm 0.1$ & 63 & 579 & 0.15 \\
\hline & 0.001 & $293.5 \pm 3.4$ & $224.9 \pm 2.8$ & $-30.2 \pm 1.0$ & $65.2 \pm 0.2$ & $42.0 \pm 0.1$ & 63 & 80 & 0.12 \\
\hline \multirow[t]{2}{*}{20} & 0.00001 & $296.2 \pm 6.7$ & $228.4 \pm 6.0$ & $-31.5 \pm 2.1$ & $65.0 \pm 0.4$ & $42.1 \pm 0.2$ & 63 & 1364 & 0.29 \\
\hline & 0.001 & $295.2 \pm 6.9$ & $227.3 \pm 6.1$ & $-31.1 \pm 2.2$ & $65.1 \pm 0.4$ & $42.1 \pm 0.2$ & 63 & 537 & 0.23 \\
\hline \multirow[t]{2}{*}{40} & 0.00001 & $297.5 \pm 8.9$ & $229.6 \pm 8.1$ & $-32.1 \pm 2.7$ & $65.0 \pm 0.4$ & $42.1 \pm 0.2$ & 63 & 745 & 0.27 \\
\hline & 0.001 & $301.0 \pm 10.1$ & $232.9 \pm 9.4$ & $-32.9 \pm 3.1$ & $65.2 \pm 0.5$ & $42.1 \pm 0.2$ & 63 & 235 & 0.34 \\
\hline \multirow[t]{5}{*}{80} & 0.00001 & $304.2 \pm 15.9$ & $236.4 \pm 15.5$ & $-34.9 \pm 4.6$ & $64.8 \pm 0.5$ & $42.2 \pm 0.2$ & 63 & 1404 & 0.50 \\
\hline & 0.001 & $305.7 \pm 13.7$ & $238.1 \pm 13.5$ & $-34.7 \pm 3.7$ & $65.0 \pm 0.5$ & $42.1 \pm 0.2$ & 63 & 734 & 0.41 \\
\hline & & shower sep & arated from $\mathrm{CA}$ & AS database il & this work & & & & \\
\hline & & $\begin{array}{l}294.8 \pm 7.4 \\
\text { shower sepa }\end{array}$ & $\begin{array}{l}227.8 \pm 6.8 \\
\text { ated from Son }\end{array}$ & $-30.2 \pm 2.8$ & $\begin{array}{c}64.8 \pm 1.7 \\
n \text { this work }\end{array}$ & $42.0 \pm 1.5$ & 62 & 10 & 0.19 \\
\hline & & $291.9 \pm 7.5$ & $224.4 \pm 6.3$ & $-30.8 \pm 2.3$ & $64.3 \pm 1.3$ & $41.8 \pm 0.8$ & 62 & 5 & 0.15 \\
\hline
\end{tabular}

Notes. $t_{\mathrm{ev}}$ - the period following the orbital evolution (the theoretical stream was modeled before this time); $\beta$ - parameter characterizing the strength of the P-R effect; Ref. - reference to the work, where the parameters of real showers were published (this reference replaces parameters $t_{\mathrm{ev}}$ and $\beta$ in the case of a real shower); $\lambda_{\odot}$ - solar longitude corresponding to the expected maximum of a shower; $\alpha$ and $\delta$ - equatorial coordinates of geocentric radiant; $V_{\mathrm{g}}$ and $V_{\mathrm{h}}-$ geocentric and heliocentric velocity; $\gamma-$ angular distance of the mean radiant from the Sun at the time corresponding to the $\lambda_{\odot}$ of maximum; $N_{\mathrm{s}}$ - the number of particles in the orbits approaching the Earth's orbit within 0.05 au or the real meteors of a given shower selected from the given database; $D_{\lim }$ - the threshold value of the Southworth-Hawkins $D$-discriminant used to separate the meteors of the filament or an observed shower. Time $t_{\mathrm{ev}}$ is given in kilo-years, the angular quantities are given in degrees, and velocities in $\mathrm{km} \mathrm{s}^{-1}$.

The agreement implies that the particles with the properties in the given model, with the given $t_{\mathrm{ev}}$ and $\beta$, are likely also present in the real stream. A more complex description of the latter is achieved as a composition of successful models.

We compare our theoretical results for the predicted showers with the corresponding observed showers selected from two International Astronomical Union (IAU) Meteor Data Center (MDC) databases: photographic, version-2013 (Porubčan et al. 2011; Neslušan et al. 2014) and Cameras for Allsky Meteor Surveillance (CAMS) video (Gural 2011; Jenniskens et al. 2011, 2016a-c; Jenniskens \& Nénon 2016). In addition, we use the 2007-2015 SonotaCo video (SonotaCo 2009, 2016), EDMOND video (Kornoš et al. 2014a,b), and radio-meteor (Hawkins 1963; Sekanina \& Southworth 1975; Lindblad, priv. comm.) databases. These databases contain 4873, 110521, 208826 , 145830 , and 62907 records on meteor orbits and geophysical data, respectively.

To identify a predicted shower and match it to its potential counterpart in a given database, we use the "break-point method" (Neslušan et al. 1995, 2013c). The method is based on an analysis of the dependence of the number of the selected meteors of a shower on the limiting value of the SH D discriminant, $D_{\lim }$, used for the selection. If a shower is present in a database, then the dependence $N=N\left(D_{\text {lim }}\right)$ has a convex behavior with a constant or almost constant part - a plateau. The break-point is a critical point in the dependence, giving the most suitable limiting value for the $D$ discriminant for the selection of the densest part of a particular shower from the near orbital phase space. 
Table 2. Mean orbital characteristics with the dispersion (characterized by standard deviation) of the predicted annual meteor showers associated with the parent body considered.

\begin{tabular}{|c|c|c|c|c|c|c|c|}
\hline$t_{\text {ev }} ;$ Ref. & $\bar{\beta}$ & $q$ & $a$ & $e$ & $\omega$ & $\bar{\Omega} \Omega$ & $i$ \\
\hline \multirow{3}{*}{5} & & Fila & nent F1 - 1? & July Pegasids & & & \\
\hline & 0.00001 & $0.547 \pm 0.022$ & $34 \pm 3$ & $0.984 \pm 0.001$ & $261.5 \pm 2.7$ & $108.1 \pm 3.2$ & $148.9 \pm 0.5$ \\
\hline & 0.001 & $0.564 \pm 0.015$ & $36 \pm 2$ & $0.984 \pm 0.001$ & $259.1 \pm 2.1$ & $105.3 \pm 2.6$ & $148.5 \pm 0.4$ \\
\hline \multirow[t]{2}{*}{10} & 0.00001 & $0.535 \pm 0.024$ & $35 \pm 3$ & $0.985 \pm 0.001$ & $264.5 \pm 3.9$ & $112.4 \pm 4.8$ & $149.3 \pm 0.5$ \\
\hline & 0.001 & $0.550 \pm 0.023$ & $36 \pm 3$ & $0.985 \pm 0.001$ & $262.2 \pm 3.3$ & $109.5 \pm 4.1$ & $149.1 \pm 0.5$ \\
\hline \multirow[t]{2}{*}{20} & 0.00001 & $0.521 \pm 0.034$ & $37 \pm 3$ & $0.986 \pm 0.002$ & $267.5 \pm 5.2$ & $116.7 \pm 6.7$ & $149.3 \pm 0.5$ \\
\hline & 0.001 & $0.536 \pm 0.030$ & $37 \pm 3$ & $0.985 \pm 0.002$ & $265.0 \pm 4.3$ & $113.8 \pm 5.6$ & $149.3 \pm 0.5$ \\
\hline \multirow[t]{2}{*}{40} & 0.00001 & $0.538 \pm 0.038$ & $38 \pm 4$ & $0.986 \pm 0.002$ & $265.6 \pm 5.2$ & $114.9 \pm 7.5$ & $148.6 \pm 0.7$ \\
\hline & 0.001 & $0.551 \pm 0.040$ & $37 \pm 4$ & $0.985 \pm 0.002$ & $263.5 \pm 5.3$ & $113.0 \pm 8.1$ & $148.7 \pm 1.0$ \\
\hline \multirow[t]{5}{*}{80} & 0.00001 & $0.562 \pm 0.048$ & $43 \pm 5$ & $0.987 \pm 0.002$ & $263.5 \pm 5.9$ & $111.2 \pm 11.4$ & $146.1 \pm 1.5$ \\
\hline & 0.001 & $0.578 \pm 0.055$ & $42 \pm 5$ & $0.986 \pm 0.002$ & $261.2 \pm 6.7$ & $108.6 \pm 13.1$ & $146.3 \pm 1.8$ \\
\hline & \multicolumn{7}{|c|}{ July Pegasids separated from CAMS database in this work } \\
\hline & & $0.582 \pm 0.042$ & $259 \pm 427$ & $0.961 \pm 0.054$ & $262.8 \pm 5.4$ & $110.8 \pm 5.5$ & $148.7 \pm 1.8$ \\
\hline & \multicolumn{7}{|c|}{ July Pegasids in the IAU MDC list } \\
\hline \multicolumn{2}{|c|}{ Jenniskens (2006) } & 0.536 & 44 & & 267.2 & 107.5 & 131.6 \\
\hline \multicolumn{2}{|c|}{ Ueda (2012) } & 0.564 & 14.78 & 0.962 & 264.73 & 110.9 & 148.77 \\
\hline \multicolumn{2}{|c|}{ Holman \& Jenniskens (2012) } & 0.598 & & 0.883 & 260.5 & 109.1 & 149.3 \\
\hline \multicolumn{2}{|c|}{ Jenniskens et al. (2016a) } & 0.562 & 7.26 & 0.960 & 265.0 & 112.4 & 149.2 \\
\hline \multicolumn{8}{|c|}{ Southern alpha Pegasids in the IAU MDC list } \\
\hline \multirow{2}{*}{\multicolumn{2}{|c|}{ Andreić et al. (2013) }} & 0.564 & 16 & 0.964 & 265 & 112 & 148.8 \\
\hline & & \multicolumn{6}{|c|}{ July gamma Pegasids in the IAU MDC list } \\
\hline \multicolumn{2}{|c|}{ Rudawska \& Jenniskens (2014) } & 0.524 & & 0.904 & 273.8 & 120.8 & 150.0 \\
\hline \multicolumn{2}{|c|}{ Kornoš et al. (2014b) } & 0.484 & & 0.922 & 275.2 & 120.5 & 149.4 \\
\hline \multirow{4}{*}{5} & & & Filam & t F2 & & & \\
\hline & 0.00001 & $0.476 \pm 0.017$ & $37 \pm 3$ & $0.987 \pm 0.001$ & $262.5 \pm 1.5$ & $109.2 \pm 1.8$ & $149.4 \pm 0.1$ \\
\hline & 0.0001 & $0.478 \pm 0.011$ & $37 \pm 2$ & $0.987 \pm 0.001$ & $262.2 \pm 1.1$ & $108.9 \pm 1.3$ & $149.5 \pm 0.1$ \\
\hline & 0.001 & - & - & - & - & - & - \\
\hline \multirow[t]{2}{*}{10} & 0.00001 & $0.491 \pm 0.026$ & $37 \pm 5$ & $0.986 \pm 0.002$ & $267.0 \pm 4.3$ & $115.3 \pm 5.2$ & $149.7 \pm 0.2$ \\
\hline & 0.001 & $0.476 \pm 0.024$ & $43 \pm 5$ & $0.989 \pm 0.002$ & $263.3 \pm 3.0$ & $110.3 \pm 3.7$ & $149.6 \pm 0.2$ \\
\hline \multirow[t]{2}{*}{20} & 0.00001 & $0.476 \pm 0.038$ & $42 \pm 8$ & $0.988 \pm 0.003$ & $266.4 \pm 5.9$ & $115.3 \pm 7.8$ & $149.5 \pm 0.5$ \\
\hline & 0.001 & $0.474 \pm 0.040$ & $44 \pm 8$ & $0.989 \pm 0.003$ & $265.4 \pm 6.0$ & $113.7 \pm 8.0$ & $149.5 \pm 0.4$ \\
\hline \multirow[t]{2}{*}{40} & 0.00001 & $0.482 \pm 0.047$ & $45 \pm 10$ & $0.989 \pm 0.003$ & $267.4 \pm 6.8$ & $116.7 \pm 9.9$ & $148.6 \pm 0.9$ \\
\hline & 0.001 & $0.498 \pm 0.055$ & $43 \pm 11$ & $0.988 \pm 0.004$ & $269.1 \pm 7.7$ & $120.1 \pm 11.2$ & $148.8 \pm 1.2$ \\
\hline \multirow[t]{6}{*}{80} & 0.00001 & $0.512 \pm 0.073$ & $50 \pm 16$ & $0.989 \pm 0.004$ & $271.2 \pm 8.9$ & $123.6 \pm 16.3$ & $145.5 \pm 2.7$ \\
\hline & 0.001 & $0.509 \pm 0.062$ & $47 \pm 13$ & $0.988 \pm 0.004$ & $270.7 \pm 8.1$ & $125.1 \pm 14.4$ & $146.8 \pm 2.1$ \\
\hline & & & ed from $\mathrm{CA}$ & US databa & s work & & \\
\hline & & $0.457 \pm 0.063$ & $308 \pm 477$ & $0.982 \pm 0.063$ & $265.1 \pm 8.4$ & $114.8 \pm 7.4$ & $151.9 \pm 3.9$ \\
\hline & & shower separat & d from Son & aCo database in & his work & & \\
\hline & & $0.456 \pm 0.051$ & $35 \pm 37$ & $0.971 \pm 0.031$ & $264.8 \pm 6.4$ & $111.9 \pm 7.5$ & $148.5 \pm 3.6$ \\
\hline
\end{tabular}

Notes. $t_{\mathrm{ev}}, \beta$, and Ref. - as in Table $1 ; q$ - perihelion distance; $a$ - semi-major axis; $e$ - eccentricity; $\omega$ - argument of perihelion; $\Omega$ - longitude of ascending node; and $i$-inclination to the ecliptic. Quantities $q$ and $a$ are given in astronomical units and angular elements in degrees.

Recently, Moorhead (2016) suggested a similar method for isolating meteor showers from the sporadic background, emphasizing that a cut-off value chosen has to reflect the strength of the shower compared to the local sporadic background. This demand is satisfied by the break-point method.

Within the break-point method, one considers all meteors from an used database and calculates their D discriminant with respect to assumed mean orbit. All meteors with $D \leq D_{\lim }$ are selected and a new mean orbit is determined. This is done as an iteration process, which is finished when the given mean orbit negligibly differs from the previous mean orbit. In the beginning, one needs an initial mean orbit. In our approach, we consider all predicted mean orbits of all filaments as the initial orbits entering the iteration.

\section{The predicted showers}

In the first stage, we model a theoretical stream of C/1979 Y1 for all combinations of time $t_{\mathrm{ev}}$ equal to $5,10,20,40$, and $80 \mathrm{kyr}$ and P-R-effect parameter $\beta=0.00001,0.0001$, and 0.001. It appears that the modeled stream crosses the Earth's orbit in two filaments, labeled as F1 and F2, which correspond to two potential showers of the comet, one before (F1) and one after (F2) perihelion (see Sect. 4.2 later). In a further process, we model the streams also for various higher values of parameter $\beta$. The mean geophysical parameters of all the filaments in the models with low $\beta$-values are given in Table 1 and their mean orbital elements can be seen in Table 2 .

After selecting the particles approaching the Earth's orbit within the chosen limit, $0.05 \mathrm{au}$, we get a set of particles that are mixed from all the filaments. The separation of particles from the individual filaments is done on the basis of the different positions of their radiant areas. The areas of filaments F1 and F2 are defined to lay in the part of the sky delimited by

F1: $\left(\alpha>300^{\circ}\right.$ or $\left.\alpha<90^{\circ}\right)$ and $\delta>0^{\circ}$;

$\mathrm{F} 2: 175^{\circ}<\alpha \leq 300^{\circ}$ and $\delta<0^{\circ}$,

where $\alpha$ and $\delta$ are equatorial coordinates of the geocentric radiant. F1 (F2) is situated northward (southward) from the ecliptic. 


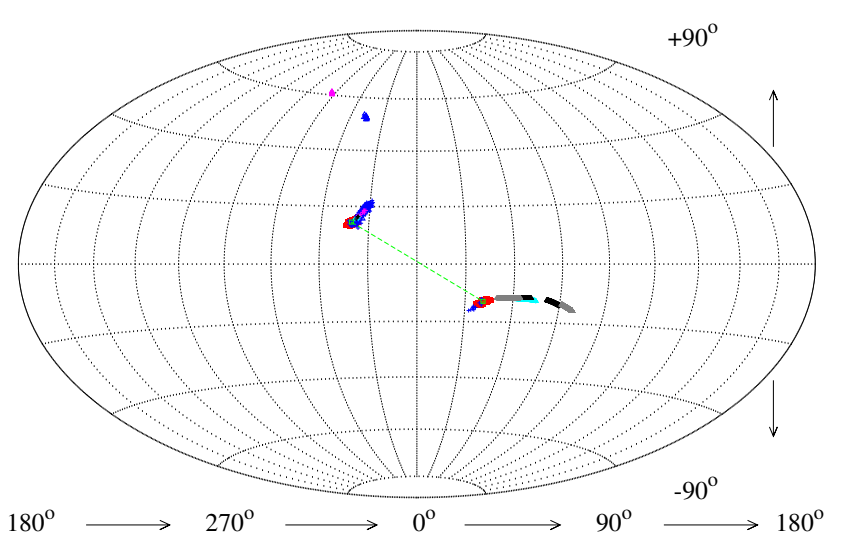

Fig. 4. Positions of radiants of theoretical particles in filaments F1, F2 (red full squares), and transitory filaments crossing the Earth's orbit. For a better transparency, the radiants of filaments F1 and F2 are shown only in the model for $t_{\mathrm{ev}}=10 \mathrm{kyr}$ and $\beta=0.00001$, and radiants of transitory filaments for all considered $t_{\mathrm{ev}}$, but only for $\beta=0.0045$ (crosses) and 0.006 (empty triangles; the symbols can be distinguished when the image is magnified on a computer screen). In the case of transitory filaments, the individual colors - blue, violet, cyan, black, and gray - distinguish between the radiants in the models for $t_{\mathrm{ev}}=80,40,20,10$, and $5 \mathrm{kyr}$, respectively. The mean radiants of filaments F1 and F2 are shown by the green empty squares and are connected with the green dashed abscissa passing through the coordinate origin. The radiants are shown in the modified ecliptical coordinate frame with the center in the apex of the Earth's motion.

The areas are in the vicinity of the ecliptic; therefore, the corresponding showers can be classified as ecliptical.

The areas of the filaments F1 and F2, which consist of particles that were not significantly influenced by the nongravitational P-R effect, are symmetric in respect to the apex of the Earth's motion around the Sun. This fact can be seen in Fig. 4, where the radiants of the theoretical particles of these two filaments, as well as other filaments discussed below, are plotted in the Hammer projection of the celestial sphere. In the figure, the modified ecliptical coordinate system is used. In contrast to the common ecliptical coordinates, the ecliptical longitude, $\lambda$, is replaced with the coordinate $\lambda_{2}$ related to the latter and the solar longitude $\lambda_{\odot}$ as

$\lambda_{2}=\lambda-\left(\lambda_{\odot}+270^{\circ}\right)$.

Concerning the radiant areas of so-called transitory filaments (Sect. 4.3), it appears that, in a specific model, only a single filament occurs in the first and/or second parts of the sky delimited by

TF1: $180^{\circ}<\alpha \leq 300^{\circ}$ and $\delta>0^{\circ}$;

TF2: $\alpha \leq 175^{\circ}$ and $\delta<0^{\circ}$.

A more detailed description of the individual filaments is given in the following subsections.

\subsection{Filament F1 - 175 July Pegasids}

Meteors in filament F1 are predicted to be relatively numerous (see the penultimate column in Table 1) and the filament has a prolonged radiant area, which can be seen in Fig. 5a. It is in the vicinity of the ecliptic. The angular distance of its mean radiant from the Sun is significantly larger than $90^{\circ}$ (see values of $\gamma$ in Table 1). Therefore, the meteors of the corresponding shower are detectable on the night sky.
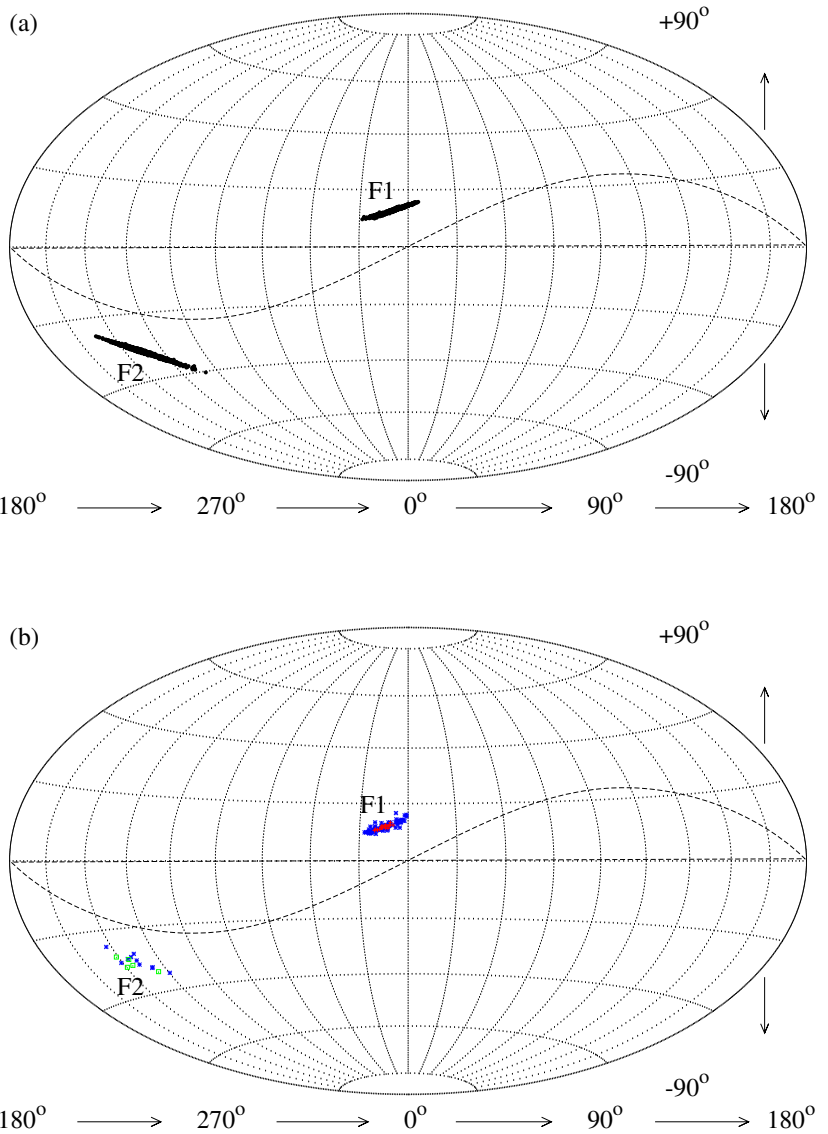

Fig. 5. Positions of radiants of theoretical particles representing the quasi-stable part (filaments F1 and F2) of the modeled stream of comet C/1979 Y1, which currently move in orbits, along which they can approach the Earth's orbit within 0.05 au (plot $a$ ). The radiant positions of the meteors of corresponding showers separated from the CAMS (red crosses for F1 and $D_{\lim }=0.07$ and blue asterisks for larger $D_{\lim }$ and both F1 and F2) and SonotaCo (green empty squares) video databases are shown in plot $b$. The shown theoretical radiants of filaments $\mathrm{F} 1$ and F2 were obtained in the models for $t_{\mathrm{ev}}=20 \mathrm{kyr}$ and $\beta=0.00001$. The positions are shown in the equatorial coordinate frame. The sinusoidlike curve illustrates the ecliptic.

In more detail, the larger particles, which are not significantly influenced by the P-R effect, tend to be in the orbits on a collisional course with the Earth. These particles are characterized by $\beta \lesssim 0.0001$. In the models for $\beta=0.00001$ and $\beta=0.0001$, there are only minute differences in the predicted mean characteristics. The P-R effect starts to be significant for $\beta \gtrsim 0.001$.

Using the break-point method (Neslušan et al. 1995, 2013c), we attempted to separate the shower corresponding to filament F1 from all the databases considered. Unfortunately, the dependence of the number of selected meteors, $N$, on the threshold Southworth-Hawkins (1963) $D$-discriminant, $D_{\text {lim }}$, does not exhibit a clear break point. For all five databases, the dependence $N=N\left(D_{\text {lim }}\right)$ is shown in Fig. 6 for F1. In the photographic data (Fig. 6a), the first meteor with an orbit similar to the mean orbit of filament F1 is separated no earlier than for $D_{\text {lim }}=0.19$. Then the number increases up to 7 for $D_{\lim }=0.27$, but this increase is steep, without any plateau. The subsequent decrease means a departure to a different phase space. Therefore, there is no pattern of a shower corresponding to F1 in these data. Such a shower is also absent in the radio data (Fig. 6e) where no meteor is separated up to the relatively high value of $D_{\lim }=0.25$, eight meteors 

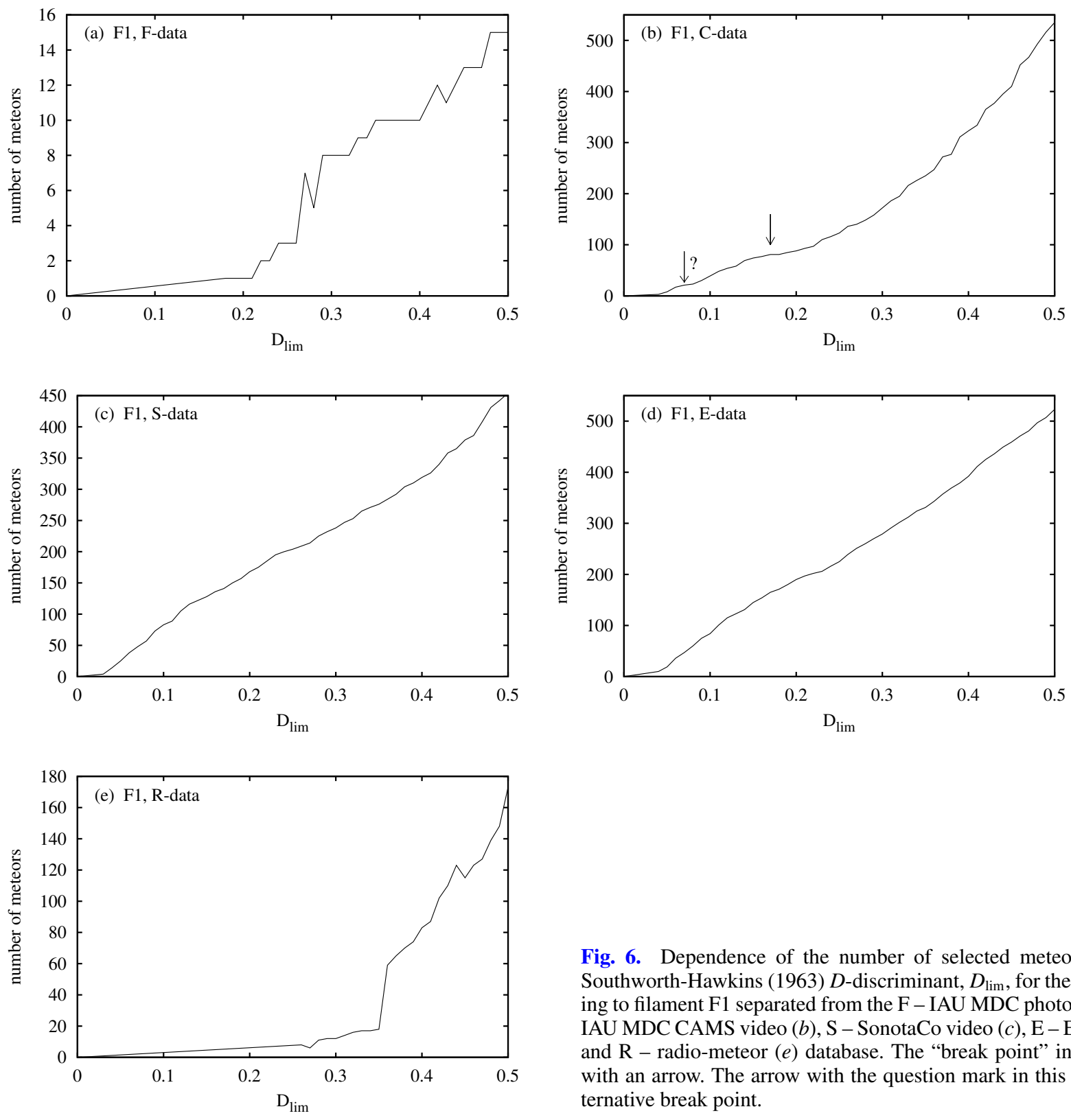

Fig. 6. Dependence of the number of selected meteors on the threshold Southworth-Hawkins (1963) $D$-discriminant, $D_{\text {lim }}$, for the shower corresponding to filament F1 separated from the F - IAU MDC photographic (plot $a$ ), $\mathrm{C}$ IAU MDC CAMS video $(b), \mathrm{S}-$ SonotaCo video $(c), \mathrm{E}-\mathrm{EDMOND}$ video $(d)$, and $\mathrm{R}$ - radio-meteor ( $e$ ) database. The "break point" in plot $b$ ) is indicated with an arrow. The arrow with the question mark in this plot indicates an alternative break point.

are separated for $D_{\text {lim }}=0.26$, but only six meteors for the subsequent value $D_{\text {lim }}=0.27$. The decrease again means a loss of the relationship of the separated group of meteors with F1.

In the SonotaCo (Fig. 6c) and EDMOND (Fig. 6d) video data, the $N=N\left(D_{\text {lim }}\right)$ curve does not have a parabola-like shape, which is typical for a sporadic background. Therefore, there are probably some meteors in orbits similar to the mean orbit of F1. However, there is no apparent plateau and so it is difficult to determine the appropriate $D_{\text {lim }}$ to separate the shower. In addition, the absence of a plateau implies a strong contamination of the phase space of the showers examined with meteors that are not its members. We would rather not separate any showers from these databases.

A certain plateau seems to only be in the $N=N\left(D_{\text {lim }}\right)$ dependence in the CAMS video data (Fig. 6b). It is situated beyond the value $D_{\text {lim }}=0.17$, which is regarded as the value corresponding to the break point and used to separate the shower. In Fig. 6b, the point concerned is indicated with an arrow. The shower consists of 81 meteors. Nonetheless, we cannot regard this separation as reliable. To check if the separation procedure provides

the meteors in the same phase space for $D_{\text {lim }}$ ranging from 0.04 (no meteors are separated for a lower $D_{\text {lim }}$ ) to 0.17 , we also consider the sub-group of 21 meteors separated for $D_{\lim }=0.07$. In Fig. $6 \mathrm{~b}$, the corresponding point is indicated with an arrow beside the question mark. The point is at the beginning of a short, alternative plateau.

The mean characteristics of the separated shower are very similar to the July Pegasids, No. 175 in the IAU MDC list of established showers (Jopek \& Kaňuchová 2014) ${ }^{2}$. Therefore, we identify filament F1 to the July Pegasids. The level of the agreement between the mean parameters predicted in this work and determined by several independent authors can be seen in Tables 1-3.

In the phase space of orbital elements, the shower occurs in the close vicinity of another established shower, 522 Southern $\alpha$-Pegasids. Andreić et al. (2013) deemed it identical to 175 July Pegasids and recommended that it be removed from the list.

2 http://www.ta3.sk/IAUC22DB/MDC2007/Roje/roje_lista. php? corobic_roje $=1 \&$ sort_roje $=0$ 
Similarly, shower No. 462 July $\gamma$-Pegasids was identified as part of the 175 July Pegasids (Rudawska \& Jenniskens 2014; Kornoš et al. 2014b) and was subsequently removed from the IAU MDC list. This might be an example of a meteor shower being alternatively named multiple times.

The radiants of the particles approaching the Earth's orbit within 0.05 au in the models for $\beta=0.00001$ and all considered evolutionary times $t_{\mathrm{ev}}$ are shown in Fig. 7a. The size of radiant area increases with increasing evolutionary time. This fact is evidence that the spatial corridor of the July Pegasids is not delimited by the resonance action of a planet. The dispersion of the shower members increases with time. Here, we observe a normal aging of the shower. Comparing the theoretical radiant areas in Fig. 7a to their real counterparts in Fig. 7b, we can state that the separated July Pegasids are likely younger than $10 \mathrm{kyr}$.

\subsection{Shower corresponding to filament F2}

The mean orbit of filament F2 is almost identical to the mean orbit of filament F1 (see Table 2), suggesting that there is only a single filament, in fact. Two potential showers are predicted because the filament of large meteoroids, not significantly influenced by the P-R effect, crosses the orbit of our planet in two positions. In this case, the denotation F1 and F2 refers to two crossing positions of one filament with the Earth's orbit rather than to two individual filaments. This is analogous to the stream of comet 1P/Halley, which also crosses the Earth's orbit twice, as $\eta$-Aquariids and Orionids. In contrast to the night showers of the $1 \mathrm{P}$ stream, the shower corresponding to $\mathrm{F} 2$ is predicted to be a daytime shower since the angular distance of its radiant from the Sun is only $\gamma \sim 63^{\circ}$ (see Table 1 ). It should be active in the middle of January.

We also attempted to separate a shower corresponding to filament F2 from all of the data sets considered. Because there are still some minute differences between the mean orbits of both F1 and F2 filaments, we did the separation for F2 again, with the initial orbits of F2 entering the iteration procedure. The behaviors of the $N=N\left(D_{\text {lim }}\right)$ dependence are shown in Fig. 8 in this case. No corresponding shower can be found in the photographic database (Fig. 8a). For values $D_{\text {lim }}<0.27$, there is no apparent plateau and a decrease occurs beyond this value. The curve in Fig. 8a can be seen to be very similar to that in Fig. 6a. This is a consequence of the fact that the mean orbit of both filaments is almost the same and the orbital phase space in the vicinity of F2 orbit is poorly filled with meteors. Therefore, the separation procedure proceeds in the same way, starting with almost the same initial orbit.

In the EDMOND video (Fig. 8d) and radio data (Fig. 8e), a plateau starts at $D_{\text {lim }}=0.14$ and 0.20 , respectively. However, only three meteors would be separated for these values of $D_{\mathrm{lim}}$. Another one or two meteors would be added for some larger values, corresponding to the middle of the plateau. Nevertheless, it would still be a relatively low number in the case of the relatively abundant EDMOND and radio data. We do not consider these sparse showers in our discussion.

A break point can be found in the $N=N\left(D_{\text {lim }}\right)$ dependence for IAU MDC CAMS (Fig. 8b) and SonotaCo (Fig. 8c) video databases. In the plots, the break points are shown with arrows. The critical value of $D_{\text {lim }}$ to separate the shower corresponding to F2 from CAMS (SonotaCo) data is $0.19(0.15)$ and the separated shower consists of ten (five) meteors. (Whether or not we should consider the sparse (five-member) shower from the large SonotaCo database was debatable. We decided to consider it because
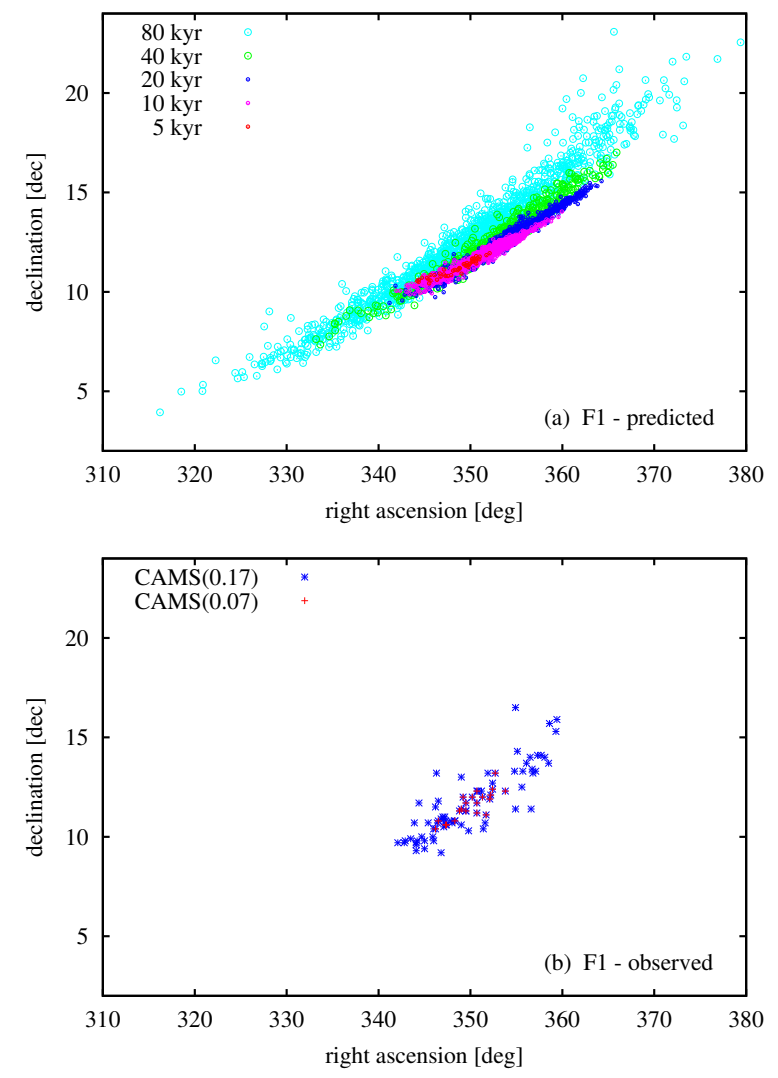

Fig. 7. Positions of radiants of theoretical particles (plot a)) of filament $\mathrm{F} 1$ and those of real meteors of the corresponding shower b). The meteors were separated from the CAMS (blue asterisks for $D_{\text {lim }}=0.17$ and red crosses for $D_{\lim }=0.07$ ) video database. The shown theoretical radiants were obtained in the models for $\beta=0.00001$ and $t_{\mathrm{ev}}=5,10,20$, 40 , and $80 \mathrm{kyr}$. The positions for the different $t_{\mathrm{ev}}$ are distinguished using the different marks and colors. The coordinate frame is equatorial.

the break point is well-defined and the shower is consistent with that obtained from the CAMS data.)

One could, perhaps, regard the points corresponding to $D_{\text {lim }}=0.31$ for CAMS and $D_{\text {lim }}=0.20$ for SonotaCo data as the alternative break points. We also separated the showers for these values of $D_{\text {lim }}$. Their mean characteristics were, however, significantly different from those for the values of $D_{\lim }=0.19$ and 0.15 , respectively, as well as from the predicted mean parameters. The iteration within the separation procedure obviously proceeded into other orbital phase space.

Matching a predicted shower to a shower in the IAU MDC list of all showers was unsuccessful. In addition, no success was achieved in matching the separated shower to the showers in the list; no shower with mean parameters similar to those for F2 can be found there. The average increase in the number of separated meteors in the part of the curve for $D_{\text {lim }}$ lower than the threshold value is only moderate, as with the CAMS database (Fig. 8b) and also with the SonotaCo data set (Fig. 8c). This is likely the reason why the shower was not separated within our automatized separation of all showers described in our earlier paper (Neslušan \& Hajduková 2017).

The mean radiant of the newly separated shower is situated near star $\alpha$ in constellation Microscopium. We suggest to name the shower $\alpha$-Microscopiids.

The positions of the radiants of the particles that constitute filament F2 in the models for $\beta=0.00001$ and all considered evolutionary times, $t_{\mathrm{ev}}$, are shown in Fig. 9a. The dispersion 
Table 3. Same as Table 1, but for the models with relatively large values of parameter $\beta$.

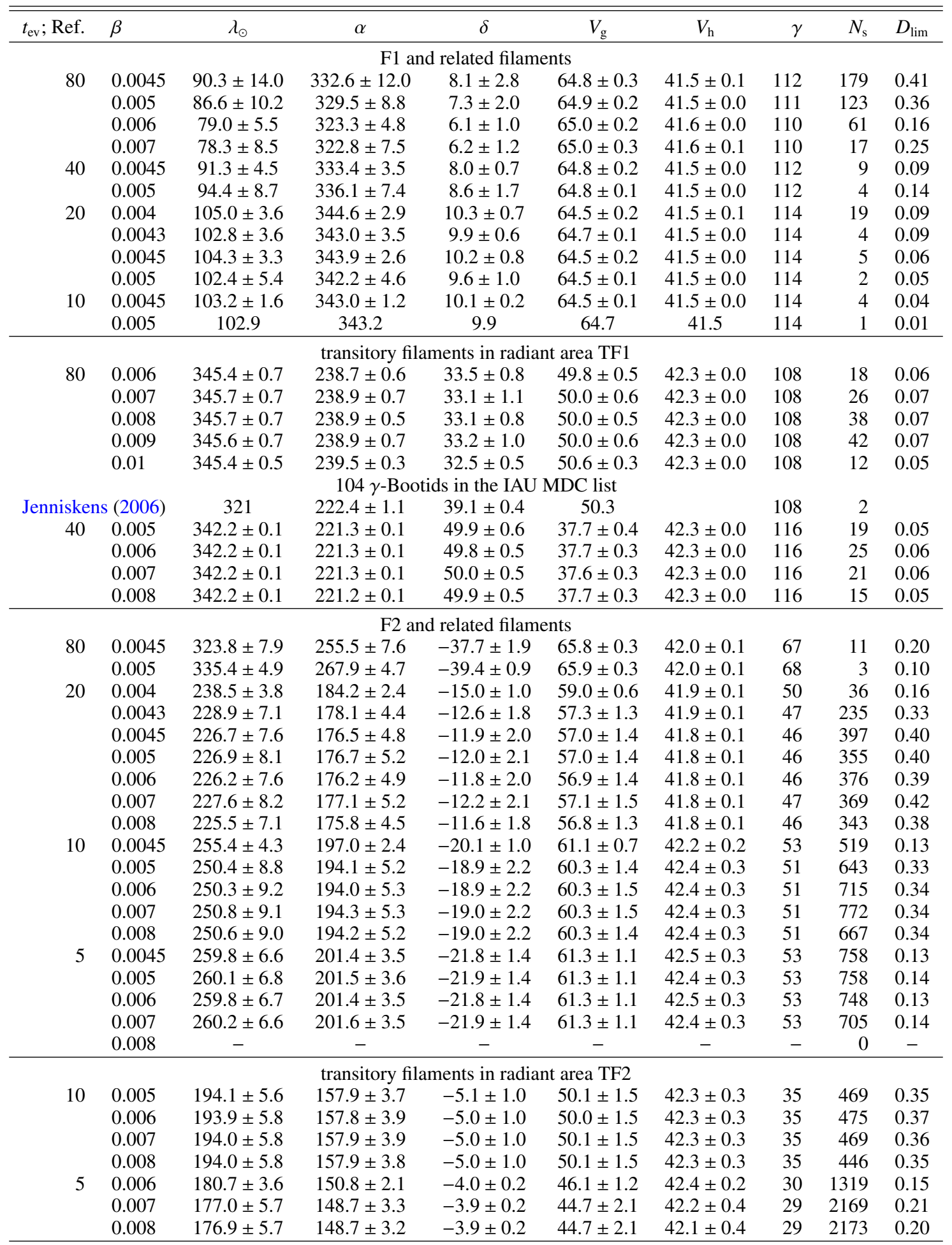

again increases with increasing time due to aging of the stream. Comparison of predicted (Fig. 9a) and observed (Fig. 9b) radiant areas confirms the age of the shower, corresponding to F2 and the whole F1-F2 filament; shorter than $10 \mathrm{kyr}$.

\subsection{The transitory filaments}

Besides the models for the low-value $\beta$-parameter, we also modeled the stream considering a set of larger $\beta$ values. Specifically, we mainly considered the values $\beta=0.0045,0.005,0.006$, $0.007,0.008$, and 0.01 . For the highest value and $t_{\mathrm{ev}} \leq 40 \mathrm{kyr}$, no shower was predicted. As expected, a stronger P-R effect deflects the particles in both F1 and F2 filaments away from the Earth's orbit and, thus, from a collisional course with our planet.

However, a complete deflection does not always occur. Sometimes, the stream is deflected away from the Earth's orbit in its section corresponding to filament F1 or F2, but it starts to cross this orbit at other sections. In contrast to filaments F1 and F2, a new filament occurs only in a model, or a few models 
Table 4. Mean radiants and the numbers of particles in the individual filaments when the P-R effect is calculated according to two different formulas.

\begin{tabular}{|c|c|c|c|c|c|c|c|c|c|}
\hline \multirow[b]{2}{*}{ fil. } & \multirow[b]{2}{*}{$\beta$} & \multicolumn{4}{|c|}{ Classical P-R formulas } & \multicolumn{4}{|c|}{ Formulas according to Klačka } \\
\hline & & $\lambda_{\odot}$ & $\alpha$ & $\delta$ & $N_{\mathrm{s}}$ & $\lambda_{\odot}$ & $\alpha$ & $\delta$ & $N_{\mathrm{s}}$ \\
\hline & \multicolumn{9}{|c|}{ Filament F1 } \\
\hline F1 & 0.00001 & $117.5 \pm 5.5$ & $354.1 \pm 4.5$ & $12.7 \pm 1.3$ & 783 & $117.6 \pm 5.6$ & $354.3 \pm 4.6$ & $12.7 \pm 1.3$ & 778 \\
\hline F1 & 0.001 & $114.7 \pm 4.9$ & $351.9 \pm 4.0$ & $12.1 \pm 1.1$ & 441 & $115.3 \pm 4.8$ & $352.4 \pm 3.9$ & $12.2 \pm 1.1$ & 443 \\
\hline F1 & 0.002 & $112.6 \pm 3.9$ & $350.3 \pm 3.2$ & $11.7 \pm 0.8$ & 194 & $112.9 \pm 4.1$ & $350.5 \pm 3.4$ & $11.8 \pm 0.9$ & 219 \\
\hline F1 & 0.003 & $109.7 \pm 3.8$ & $348.2 \pm 3.1$ & $11.1 \pm 0.8$ & 83 & $109.4 \pm 4.5$ & $347.8 \pm 3.6$ & $11.1 \pm 0.9$ & 82 \\
\hline $\mathrm{F} 1 ?$ & 0.004 & $104.9 \pm 2.9$ & $344.1 \pm 2.2$ & $10.3 \pm 0.6$ & 11 & $105.0 \pm 3.6$ & $344.6 \pm 2.9$ & $10.3 \pm 0.7$ & 19 \\
\hline \multicolumn{10}{|c|}{ Filaments F2 and transitory } \\
\hline $\mathrm{F} 2$ & 0.00001 & $295.9 \pm 6.5$ & $228.1 \pm 5.8$ & $-31.3 \pm 2.1$ & 1378 & $296.2 \pm 6.7$ & $228.4 \pm 6.0$ & $-31.5 \pm 2.1$ & 1364 \\
\hline F2 & 0.001 & $294.6 \pm 6.4$ & $226.6 \pm 5.6$ & $-30.8 \pm 2.0$ & 533 & $295.2 \pm 6.9$ & $227.3 \pm 6.1$ & $-31.1 \pm 2.2$ & 537 \\
\hline F2 & 0.002 & $293.7 \pm 6.2$ & $225.5 \pm 5.4$ & $-30.4 \pm 1.8$ & 80 & $293.8 \pm 7.2$ & $225.6 \pm 6.2$ & $-30.4 \pm 2.1$ & 94 \\
\hline F2 & 0.003 & 292.0 & 223.2 & -29.7 & 1 & $288.5 \pm 4.2$ & $220.6 \pm 3.6$ & $-28.9 \pm 1.2$ & 7 \\
\hline TF2 & 0.004 & $238.4 \pm 2.8$ & $184.1 \pm 1.8$ & $-15.0 \pm 0.7$ & 39 & $238.5 \pm 3.8$ & $184.2 \pm 2.4$ & $-15.0 \pm 1.0$ & 36 \\
\hline TF2 & 0.005 & $227.1 \pm 8.2$ & $176.8 \pm 5.2$ & $-12.0 \pm 2.1$ & 365 & $226.9 \pm 8.1$ & $176.7 \pm 5.2$ & $-12.0 \pm 2.1$ & 355 \\
\hline TF2 & 0.006 & $226.6 \pm 7.8$ & $176.5 \pm 5.0$ & $-11.9 \pm 2.0$ & 378 & $226.2 \pm 7.6$ & $176.2 \pm 4.9$ & $-11.8 \pm 2.0$ & 376 \\
\hline TF2 & 0.007 & $227.7 \pm 7.6$ & $177.2 \pm 4.8$ & $-12.2 \pm 1.9$ & 397 & $227.6 \pm 8.2$ & $177.1 \pm 5.2$ & $-12.2 \pm 2.1$ & 369 \\
\hline TF2 & 0.008 & $225.1 \pm 7.3$ & $175.5 \pm 4.7$ & $-11.5 \pm 1.9$ & 354 & $225.5 \pm 7.2$ & $175.8 \pm 4.5$ & $-11.6 \pm 1.8$ & 343 \\
\hline TF2 & 0.0083 & $222.0 \pm 7.4$ & $173.3 \pm 4.6$ & $-10.6 \pm 1.8$ & 118 & $222.4 \pm 7.8$ & $173.5 \pm 4.9$ & $-10.7 \pm 1.9$ & 125 \\
\hline
\end{tabular}

Notes. All for $t_{\mathrm{ev}}=20 \mathrm{kyr}$.


Fig. 8. Dependence of the number of selected meteors on the threshold SouthworthHawkins (1963) $D$-discriminant, $D_{\text {lim }}$, for the shower corresponding to filament F2 separated from the F - IAU MDC photographic (plot $a$ ), C - IAU MDC CAMS video $(b), \mathrm{S}$ - SonotaCo video $(c), \mathrm{E}$ - EDMOND video $(d)$, and $\mathrm{R}$ - radio-meteor (e) database. The points on the curves in plots $b$ and $c$ regarded as the "break points" are indicated by arrows. 

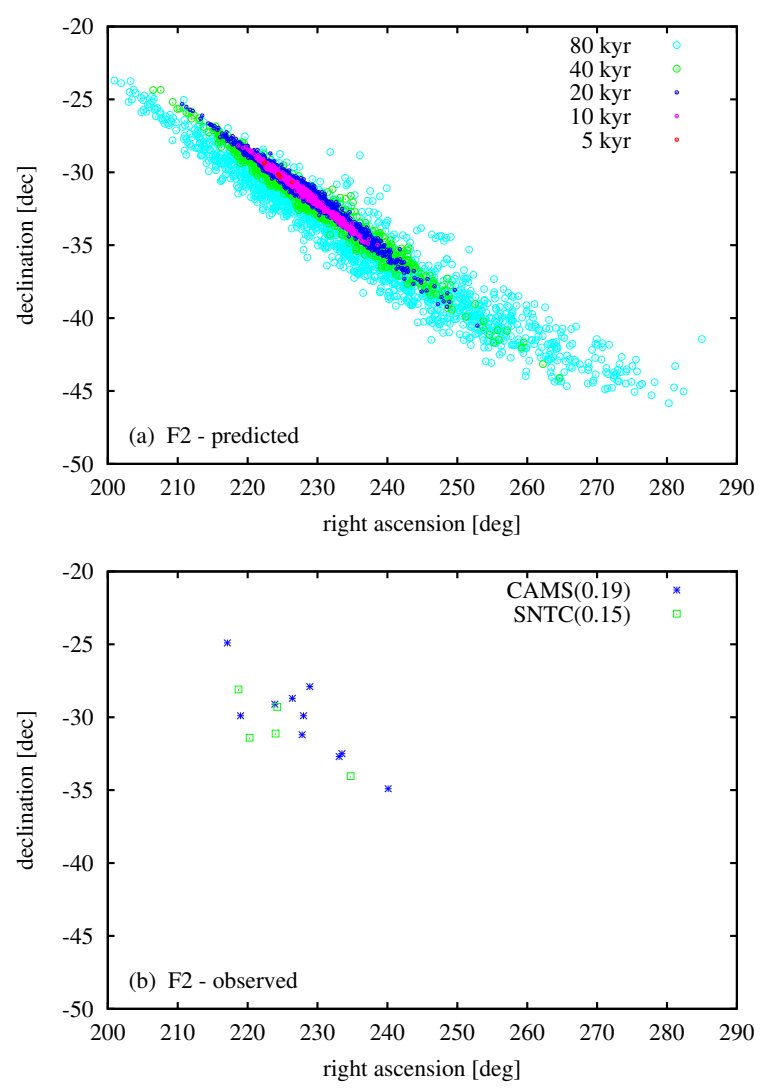

Fig. 9. Positions of radiants of theoretical particles (plot $a$ ) of filament F2 and those of real meteors in orbits resembling those of theoretical particles $(b)$. The meteors were separated from the CAMS (blue asterisks) and SonotaCo (green empty squares) video databases. The shown theoretical radiants were obtained in the models for $\beta=0.00001$ and $t_{\mathrm{ev}}=5,10,20,40$, and $80 \mathrm{kyr}$. The positions for the different $t_{\mathrm{ev}}$ are distinguished using the different marks and colors. The coordinate frame is equatorial.

with neighboring $\beta$ values, for a specific combination of $t_{\mathrm{ev}}$ and $\beta$. Therefore, it is expected to survive only during a limited period and, likely, to consist of particles that have a narrow interval of sizes. We refer to such a filament as a "transitory filament" in contrast to F1 and F2, which are predicted in almost all created models and which are regarded as annual showers.

We modeled the theoretical streams for all combinations of $t_{\mathrm{ev}}=5,10,20,40$, and $80 \mathrm{kyr}$ and $\beta=0.0045,0.005,0.006$, 0.007 , and 0.008 (in addition to values $0.00001,0.0001$, and 0.001). The transitory filaments occurred only for several combinations of $t_{\mathrm{ev}}$ and $\beta$. The mean geophysical data characterizing the occurred transitory filaments as well as filaments F1 and F2 for the large $\beta$-values are given in Table 3 . The positions of the radiants of the particles in all filaments, F1 and F2 included, are shown in Fig. 10. In more detail, a given plot illustrates the radiants for a single $t_{\mathrm{ev}}$ and all values of $\beta$, which are distinguished by the use of different colors.

The number of particles approaching the Earth's orbit within filament $\mathrm{F} 1$ decreases with increasing $\beta$-values. In the models for $t_{\mathrm{ev}}=5,10,20$, and $40 \mathrm{kyr}$, this filament disappears when $\beta$ reaches 0.005 . For the longest investigated evolutionary period of $t_{\mathrm{ev}}=80 \mathrm{kyr}$, it persists up to $\beta \sim 0.007$.

Two largely different transitory filaments occur in the radiant area TF1 for $0.005 \leq \beta \leq 0.008$ and $0.005 \leq \beta \leq 0.010$, but only after a relatively long time of the dynamical evolution of the stream, $t_{\mathrm{ev}} \geq 40 \mathrm{kyr}$. These filaments differ from each other, especially in their geocentric velocity; 50.0 versus $37.7 \mathrm{~km} \mathrm{~s}^{-1}$.

Filament F2 is also less and less populated with increasing $\beta$-values, like F1, and practically disappears at $\beta \sim 0.003$. The particles with a higher $\beta$ are obviously removed from the orbits in which they cross the Earth's orbit at its arc, which corresponds to the solar longitude $\lambda_{\odot} \sim 295^{\circ}$. At the same time, it seems that the small particles, characterized by $\beta \sim 0.005-0.008$, are moved to orbits which closely encounter the Earth's orbit at its arcs with different $\lambda_{\odot}$-values (Table 3 ). A few transitory filaments occur in the parts of the sky defined in the beginning of Sect. 4 as TF1 and TF2. The particles characterized by $\beta \gtrsim 0.01$ are completely moved into orbits in which they cannot collide with the Earth.

The chance of detecting and recording a significant number of meteors of a transitory filament is not very high. Nevertheless, we tried to separate the real showers that correspond to all found transitory filaments, represented by the number of selected particles $N_{\mathrm{s}} \geq 5$. Overall, our attempt was unsuccessful. In the case of $\mathrm{F} 1$, the separation resulted in the real showers already found in the separation for the theoretical orbits predicted for low $\beta$-values, which are given in Tables 1 and 2. Starting from the different initial orbits, the separation procedure resulted, sometimes, in a slightly different set of shower meteors, with slightly different mean parameters.

In the case of transitory filaments, the single success we achieved was an occurrence of similarity between the transitory filament with the radiant in radiant area TF1, models for $t_{\mathrm{ev}}=80 \mathrm{kyr}$, and shower $104 \gamma$-Bootids in the IAU MDC list of all showers (Table 3). In the list, this shower is not classified as an established shower, and it is currently represented by only two meteors (see Table 7, \#104, page 695 in Jenniskens 2006). Moreover, its existence was not confirmed in a recent study by Jenniskens et al. (2016b). Nevertheless, the predicted parameters agree well with their observed counterparts, except for relatively large differences in the declination of radiant, solar longitude, and, hence, longitude of ascending node. The predicted longitudes are larger (about $24^{\circ}$ ) and predicted declination is about $6^{\circ}$ lower than observed. It is possible that a better match would occur for other evolutionary times, $t_{\mathrm{ev}}$.

The transitory filaments do not exhibit symmetry in respect to the apex of the Earth motion (Fig. 4), in contrast to filaments F1 and F2.

To see a difference in the dynamics of a stream that is influenced by the P-R effect calculated by the new, improved formulas by Klačka and by the old formulas (the new formulas (2) and (3) are reduced to old ones putting $\eta_{1}=\eta_{2}=0$ ), we calculated the evolution of the modeled streams listed in Table 4 by using both sets of formulas. The result, according to the old, classical (new, Klačka's) formulas is given in Cols. 3-6 (7-10) of the table. We observe only small differences. This is not surprising since the dominant term of the P-R effect is the first term, $\beta G M_{\odot} / r^{2}$, of the radial component of the acceleration due to this effect and this term is the same in both old and new formulas. Nonetheless, the result is not identical. Therefore, the use of the new formulas is advantageous.

\section{Conclusions}

We modeled a theoretical stream of comet C/1979 Y1 (Bradfield). The models are characterized by a variety of values of free parameters, evolutionary time, and strength of the PoyntingRobertson drag $\beta$, and were used to predict a part or parts of the stream that can collide with the Earth. We aimed to predict the meteor shower(s) of the comet. Our modeling was successful, 

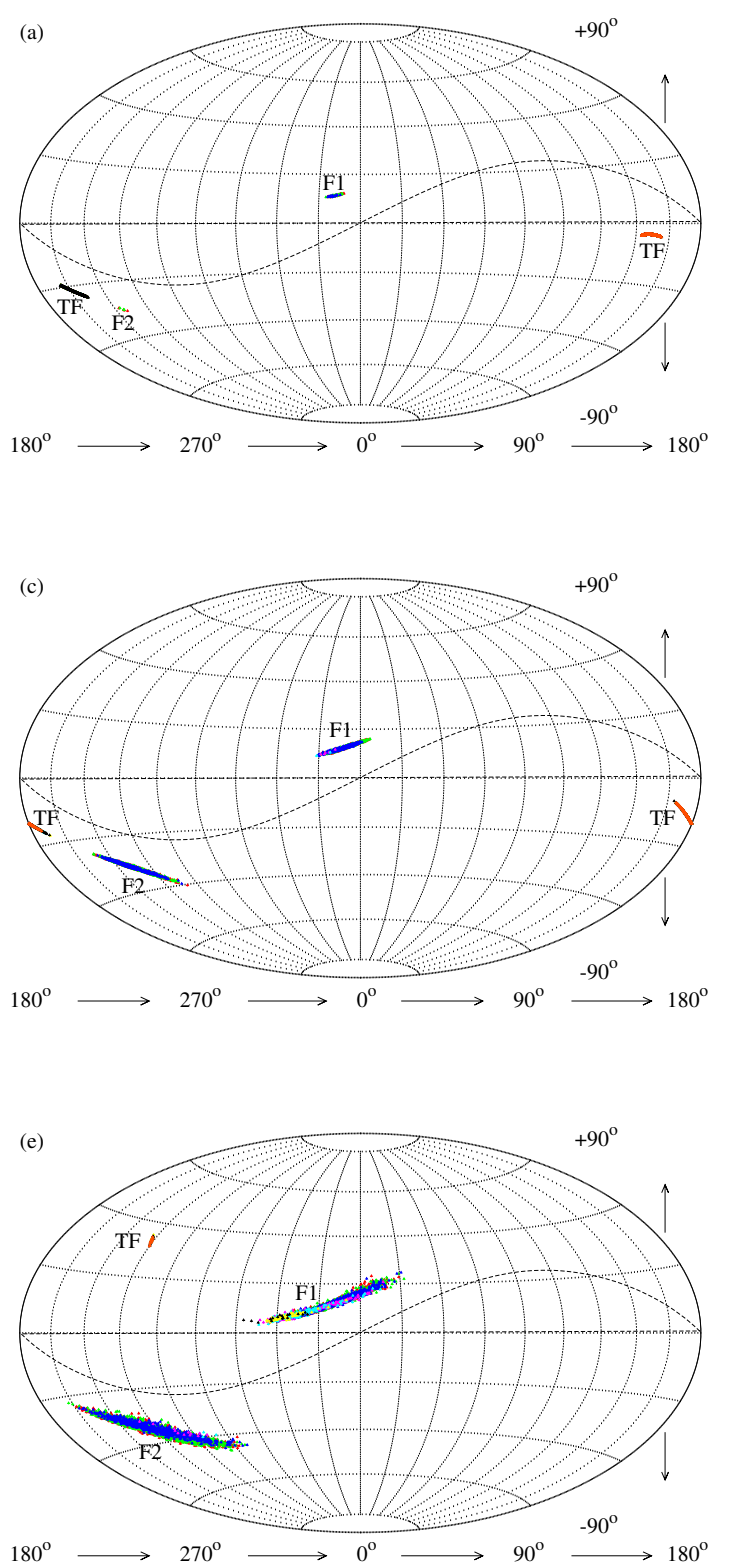
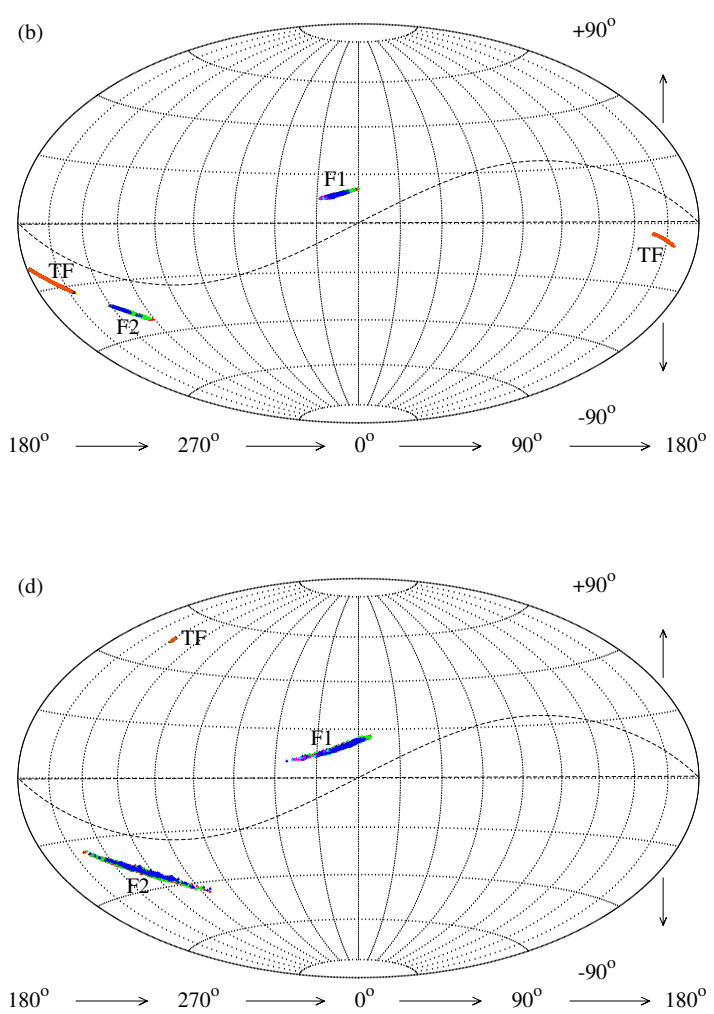

Fig. 10. Positions of theoretical radiants predicted with the models of $\mathrm{C} / 1979$ Y1-stream for $t_{\mathrm{ev}}=5($ plot $a), 10(b), 20(c), 40(d)$, and $80 \mathrm{kyr}(e)$. Filaments F1 and F2 corresponding to the annual showers, as well as the transitory filaments TF, are plotted. The positions are shown in the Hammer projection of celestial sphere in the equatorial coordinates. The sinusoid-like curve is the ecliptic.

and we were therefore able, subsequently, to separate some corresponding showers from the considered databases of real meteors and compare the prediction with the observation. Also, we searched for the corresponding showers in the IAU MDC list of showers. A comparison between the predicted showers and their real existing counterparts enabled us to identify the values of the free parameters that are relevant to the real showers.

Specifically, we found, in an agreement with other researchers, that the comet is the parent body of the established shower 175 July Pegasids. Further, we found that it is the parent body of a new daytime shower, which we named $\alpha$ Microscopiids. In addition, the comet is likely the parent body of $104 \gamma$-Bootids given in the IAU MDC list of all showers. The characteristics of this shower were, however, derived from only two observed meteors (Jenniskens 2006, Table 7, \#104 on page 695), therefore its existence is still questionable.

The July Pegasids are predicted by the models with the particles characterized by a wide range of values of the P-R-effect parameter $\beta$, from practically zero to about 0.007 . On the contrary, the transitory $\gamma$-Bootids have to consist of particles in a narrow size interval, corresponding to the values of $\beta$ ranging from $\sim 0.006$ to $\sim 0.010$. Assuming a mean density of the particles equal to $1000 \mathrm{~kg} \mathrm{~m}^{-3}$, their mean radii should span from $\sim 60$ to $\sim 100 \mu \mathrm{m}$. Furthermore, the $\gamma$-Bootids had to be released from the parent body a long time ago; $\sim 80 \mathrm{kyr}$. This is an acceptably long time, and corresponds to only about 260 orbital revolutions of the parent comet around the Sun. We remind readers that the active phase, as the parent body of a meteoroid stream, of the other long-period comet, 1P/Halley, is estimated to have already made approximately 2300 orbital revolutions ( $\sim 175 \mathrm{kyr})$ (Hajduk 1987).

While the relatively large meteoroids, which are not significantly influenced by the P-R effect, collide with the Earth in filaments F1 and F2, with the radiant areas that are situated symmetrically with respect to the Earth's apex, smaller meteoroids are predicted to collide with our planet in the filaments with radiants that do not possess any such symmetry. The filaments with asymmetric radiant areas are, obviously, those that occur exclusively due to the action of the P-R effect.

Our study of the stream of C/1979 Y1 confirms the important role of the P-R effect in the dynamical evolution of meteoroid streams. We learnt that, due to this effect, the stream can be so 
variable that some showers associated with a considered parent body might not be revealed in a study which included only the gravitational perturbations.

Acknowledgements. This article was supported by the realization of the Project ITMS No. 26220120029, based on the supporting operational Research and development program financed from the European Regional Development Fund. The work was also supported, in part, by the VEGA - the Slovak Grant Agency for Science, grants No. 2/0031/14 and 1/0225/14, and by the Slovak Research and Development Agency under the contract No. APVV-0517-12.

\section{References}

Abedin, A., Spurný, P., Wiegert, P., et al. 2015, Icarus, 261, 100

Abedin, A., Wiegert, P., Pokorný, P., \& Brown, P. 2017, Icarus, 281, 417

Andreić, Ž., Šegon, D., Korlević, K., et al. 2013, WGN, J. International Meteor Organization, 41, 103

Asher, D. J. 2008, Earth Moon and Planets, 102, 27

Asher, D. J., \& Emel'yanenko, V. V. 2002, MNRAS, 331, 126

Babadzhanov, P. B., Williams, I. P., \& Kokhirova, G. I. 2008, MNRAS, 386 2271

Babadzhanov, P. B., Williams, I. P., \& Kokhirova, G. I. 2013, A\&A, 556, A25

Babadzhanov, P. B., Kokhirova, G. I., \& Khamroev, U. K. 2015a, Adv. Space Res., 55, 1784

Babadzhanov, P. B., Kokhirova, G. I., \& Obrubov, Y. V. 2015b, A\&A, 579, A119

Chambers, J. E. 1999, MNRAS, 304, 793

Chernitsov, A. M., Baturin, A. P., \& Tamarov, V. A. 1998, Sol. Syst. Res., 32, 405

Everhart, E. 1985, in Dynamics of Comets: Their Origin and Evolution, Proceedings of IAU Colloq., 83, held in Rome, Italy, June 11-15, 1984, eds. A. Carusi, \& G. B. Valsecchi (Dordrecht: Reidel), Astrophys. Space Sci. Lib. 115,185

Giorgini, J. D., Yeomans, D. K., Chamberlin, A. B., et al. 1996, in AAS/Division for Planetary Sciences Meeting Abstracts \#28, BAAS, 28, 1158

Gural, P. S. 2011, in Proc. International Meteor Conference, 29th IMC, Armagh, Northern Ireland, 2010, 28

Hajduk, A. 1987, A\&A, 187, 925

Hawkins, G. S. 1963, Smithsonian Contributions to Astrophysics, 7, 53

Holman, D., \& Jenniskens, P. 2012, WGN, J. International Meteor Organization, 40,166

Jakubík, M., \& Neslušan, L. 2015, MNRAS, 453, 1186

Jenniskens, P. 2006, Meteor Showers and their Parent Comets (Cambridge, UK: Cambridge University Press)

Jenniskens, P., \& Nénon, Q. 2016, Icarus, 266, 371

Jenniskens, P., \& Vaubaillon, J. 2010, AJ, 139, 1822

Jenniskens, P., Gural, P. S., Dynneson, L., et al. 2011, Icarus, 216, 40

Jenniskens, P., Nénon, Q., Albers, J., et al. 2016a, Icarus, 266, 331

Jenniskens, P., Nénon, Q., Gural, P. S., et al. 2016b, Icarus, 266, 355

Jenniskens, P., Nénon, Q., Gural, P. S., et al. 2016c, Icarus, 266, 384

Jopek, T. J., \& Kaňuchová, Z. 2014, Meteoroids 2013, 353

Jopek, T. J., \& Williams, I. P. 2013, MNRAS, 430, 2377
Kasuga, T., \& Jewitt, D. 2015, AJ, 150, 152

Kaňuchová, Z., \& Neslušan, L. 2007, A\&A, 470, 1123

Klačka, J. 2014, MNRAS, 443, 213

Kokhirova, G. I., \& Babadzhanov, P. B. 2015, Meteoritics and Planetary Science, 50,461

Kornoš, L., Koukal, J., Piffl, R., \& Tóth, J. 2014a, in Proc. International Meteor Conference, Poznan, Poland, 22-25 August 2013, eds. M. Gyssens, P. Roggemans, \& P. Zoladek, 23

Kornoš, L., Matlovič, P., Rudawska, R., et al. 2014b, Meteoroids 2013, 225

Kornoš, L., Tóth, J., Porubčan, V., et al. 2015, Planet. Space Sci., 118, 48

Lyytinen, E., \& Jenniskens, P. 2003, Icarus, 162, 443

Micheli, M., Tholen, D. J., \& Jenniskens, P. 2016, Icarus, 267, 64

Molau, S., \& Rendtel, J. 2009, WGN, J. International Meteor Organization, 37, 98

Moorhead, A. V. 2016, MNRAS, 455, 4329

Neslušan, L. 1999, A\&A, 351, 752

Neslušan, L., \& Hajduková, M. 2014, A\&A, 566, A33

Neslušan, L., \& Hajduková, M. 2017, A\&A, 598, A40

Neslušan, L., Svoreň, J., \& Porubčan, V. 1995, Earth Moon and Planets, 68, 427

Neslušan, L., Hajduková, M., \& Jakubík, M. 2013a, A\&A, 560, A47

Neslušan, L., Kaňuchová, Z., \& Tomko, D. 2013b, A\&A, 551, A87

Neslušan, L., Svoreň, J., \& Porubčan, V. 2013c, Earth Moon and Planets, 110, 41

Neslušan, L., Porubčan, V., \& Svoreň, J. 2014, Earth Moon and Planets, 111, 105 Porubčan, V., Svoreň, J., Neslušan, L., \& Schunova, E. 2011, in Meteoroids: The Smallest Solar System Bodies, eds. W. J. Cooke, D. E. Moser, B. F. Hardin, \& D. Janches, 338

Rendtel, J., Arlt, R., Koschak, R., et al. 1995, in Handbook for visual meteor observers, eds. J. Rendtel, R. Arlt, \& A. McBeath (Potsdam, Germany: International Meteor Organization), 169

Rudawska, R., \& Jenniskens, P. 2014, Meteoroids 2013, 217

Rudawska, R., \& Vaubaillon, J. 2015, Planet. Space Sci., 118, 25

Ryabova, G. O. 2007, MNRAS, 375, 1371

Ryabova, G. O. 2016, MNRAS, 456, 78

Sekanina, Z., \& Southworth, R. B. 1975, Physical and dynamical studies of meteors. Meteor-fragmentation and stream-distribution studies, Tech. rep.

Sekhar, A., \& Asher, D. J. 2013, MNRAS, 433, L84

Sekhar, A., \& Asher, D. J. 2014a, MNRAS, 437, L71

Sekhar, A., \& Asher, D. J. 2014b, Meteoritics and Planetary Science, 49, 52

SonotaCo 2009, WGN, J. International Meteor Organization, 37, 55

SonotaCo 2016, WGN, J. International Meteor Organization, 44, 42

Tomko, D., \& Neslušan, L. 2012, Earth Moon and Planets, 108, 123

Tomko, D., \& Neslušan, L. 2016, A\&A, 592, A107

Ueda, M. 2012, WGN, J. International Meteor Organization, 40, 59

Vaubaillon, J., \& Jenniskens, P. 2007, in BAAS, AAS/Division for Planetary Sciences Meeting Abstracts \#39, 535

Vaubaillon, J., Arlt, R., Shanov, S., Dubrovski, S., \& Sato, M. 2005a, MNRAS, 362, 1463

Vaubaillon, J., Colas, F., \& Jorda, L. 2005b, A\&A, 439, 761

Vereš, P., Kornoš, L., \& Tóth, J. 2011, MNRAS, 412, 511

Wiegert, P. A., Brown, P. G., Vaubaillon, J., \& Schijns, H. 2005, MNRAS, 361, 638 